\title{
SCHWARZ-PICK AND LANDAU TYPE THEOREMS FOR SOLUTIONS TO THE DIRICHLET-NEUMANN PROBLEM IN THE UNIT DISK
}

\author{
PEIJIN LI, QINGHONG LUO, AND SAMINATHAN PONNUSAMY
}

\begin{abstract}
The aim of this paper is to establish some properties of solutions to the Dirichlet-Neumann problem: $\left(\partial_{z} \partial_{\bar{z}}\right)^{2} w=g$ in the unit disc $\mathbb{D}, w=\gamma_{0}$ and $\partial_{\nu} \partial_{z} \partial_{\bar{z}} w=\gamma$ on $\mathbb{T}$ (the unit circle), $\frac{1}{2 \pi i} \int_{\mathbb{T}} w_{\zeta \bar{\zeta}}(\zeta) \frac{d \zeta}{\zeta}=c$, where $\partial_{\nu}$ denotes differentiation in the outward normal direction. More precisely, we obtain Schwarz-Pick type inequalities and Landau type theorem for solutions to the Dirichlet-Neumann problem.
\end{abstract}

\section{INTRODUCTION}

Let $\mathbb{C} \cong \mathbb{R}^{2}$ denote the complex plane and for $r>0$, let $\mathbb{D}_{r}=\{z \in \mathbb{C}:|z|<r\}$. Denote by $\mathbb{D}:=\mathbb{D}_{1}$, the open unit disk, $\mathbb{T}=\partial \mathbb{D}$, the boundary of $\mathbb{D}$, and $\overline{\mathbb{D}}=\mathbb{D} \cup \mathbb{T}$, the closure of $\mathbb{D}$. Denote by $\mathcal{C}(\Omega)$, the set of all continuous functions in a domain $\Omega$ in $\mathbb{C}$. The space of integrable functions in $\Omega$ is denoted by $L^{1}(\Omega)$. Denote by $\mathcal{H}(\mathbb{D}, \mathbb{D})$ (resp. $\mathcal{A}(\mathbb{D}, \mathbb{D}))$ the class of all complex-valued harmonic (resp. analytic) self-mappings of the unit disk $\mathbb{D}$

The Dirichlet and the Neumann boundary value problems in complex analysis have been very well studied in the literature. See [2, 3] for investigations of basic boundary value problems with different kinds of boundary conditions.

In this paper we investigate some properties of solutions to the following DirichletNeumann problem:

$$
\begin{cases}\left(\partial_{z} \partial_{\bar{z}}\right)^{2} w=g & \text { in } \mathbb{D} \\ w=\gamma_{0} & \text { on } \mathbb{T} \\ \partial_{\nu} \partial_{z} \partial_{\bar{z}} w=\gamma & \text { on } \mathbb{T}\end{cases}
$$

and

$$
\frac{1}{2 \pi i} \int_{\mathbb{T}} w_{\zeta \bar{\zeta}}(\zeta) \frac{d \zeta}{\zeta}=c
$$

where $\partial_{\nu}$ denotes differentiation in the outward normal direction, $g \in L^{1}(\mathbb{D}), \gamma_{0}$, $\gamma \in C(\mathbb{T}), c \in \mathbb{C}$ is a constant, and satisfying the condition

$$
\frac{1}{2 \pi} \int_{0}^{2 \pi} \gamma\left(e^{i t}\right) d t=\frac{2}{\pi} \int_{\mathbb{D}} g(\zeta) d A(\zeta) .
$$

2010 Mathematics Subject Classification. Primary: 30C62, 31A30, 30C80; Secondary: 30C20, 31A05.

Key words and phrases. Dirichlet-Neumann problem, Schwarz's Lemma, Landau type theorem and univalent function. 
Here

$$
\partial_{z}:=\frac{\partial}{\partial z}=\frac{1}{2}\left(\frac{\partial}{\partial x}-i \frac{\partial}{\partial y}\right) \text { and } \partial_{\bar{z}}:=\frac{\partial}{\partial \bar{z}}=\frac{1}{2}\left(\frac{\partial}{\partial x}+i \frac{\partial}{\partial y}\right)
$$

represent the complex differential operators so that $\Delta=4 \partial_{z} \partial_{\bar{z}}$, is the Laplacian. So, $\partial_{z} \partial_{\bar{z}}$ and $\left(\partial_{z} \partial_{\bar{z}}\right)^{2}$ are called the harmonic and biharmonic operators, respectively. Consequently, $\partial_{z} \partial_{\bar{z}} w=0$ in $\mathbb{D}$ is equivalent to the statement that $w$ is harmonic, while a solution to the equation $\left(\partial_{z} \partial_{\bar{z}}\right)^{2} w=0$ is called a biharmonic function. See [9, 10] and the references therein for certain properties of biharmonic functions.

For $z \in \mathbb{D}$, let

$$
P\left(z, e^{i t}\right)=\frac{1-|z|^{2}}{\left|1-z e^{-i t}\right|^{2}}
$$

denote the Poisson kernel in $\mathbb{D}$. The function $z \mapsto P\left(z, e^{i t}\right)$ is harmonic in $\mathbb{D}$.

In [3], it was shown that the condition (1.3) ensures that all solutions to (1.1) satisfying the condition (1.2) are given by the formula

$$
w(z)=-c\left(1-|z|^{2}\right)+\mathcal{P}_{\gamma_{0}}(z)+\mathcal{G}_{1}[\gamma](z)-\mathcal{G}_{2}[g](z),
$$

where

$$
\begin{gathered}
\mathcal{P}_{\gamma_{0}}(z)=\frac{1}{2 \pi} \int_{0}^{2 \pi} P\left(z, e^{i t}\right) \gamma_{0}\left(e^{i t}\right) d t \\
\mathcal{G}_{1}[\gamma](z)=\frac{1}{4 \pi} \int_{0}^{2 \pi} H_{2}\left(z, e^{i t}\right) \gamma\left(e^{i t}\right) d t
\end{gathered}
$$

and

$$
\mathcal{G}_{2}[g](z)=\int_{\mathbb{D}} H_{2}(z, \zeta) g(\zeta) d A(\zeta)
$$

with

$$
\begin{aligned}
H_{2}(z, \zeta)= & -|\zeta-z|^{2} \log |\zeta-z|^{2} \\
& -\left(1-|z|^{2}\right)\left[4+\frac{1-z \bar{\zeta}}{z \bar{\zeta}} \log (1-z \bar{\zeta})+\frac{1-\bar{z} \zeta}{\bar{z} \zeta} \log (1-\bar{z} \zeta)\right] \\
& -\frac{(\zeta-z)(1-z \bar{\zeta})}{z} \log (1-z \bar{\zeta})-\frac{\overline{(\zeta-z)}(1-\bar{z} \zeta)}{\bar{z}} \log (1-\bar{z} \zeta),
\end{aligned}
$$

and $d A(\zeta)=(1 / \pi) d x d y$ denotes the normalized area measure in $\mathbb{D}$.

\section{Main Results}

2.1. A Schwarz type lemma. The classical Schwarz lemma states that if $f \in$ $\mathcal{A}(\mathbb{D}, \mathbb{D})$ such that $f(0)=0$, then $|f(z)| \leq|z|$ for all $z \in \mathbb{D}$. This result has been a crucial result in many branches of research for more than a hundred years. The harmonic analog of this statement states that if $f \in \mathcal{H}(\mathbb{D}, \mathbb{D})$ such that $f(0)=0$, then (Heniz [16])

$$
|f(z)| \leq \frac{4}{\pi} \arctan |z| \text { for } z \in \mathbb{D}
$$


Later, by removing the assumption $f(0)=0$, Pavlović [22, Theorem 3.6.1] established the following sharp inequality for $f \in \mathcal{H}(\mathbb{D}, \mathbb{D})$ :

$$
\left|f(z)-\frac{1-|z|^{2}}{1+|z|^{2}} f(0)\right| \leq \frac{4}{\pi} \arctan |z| \text { for } z \in \mathbb{D} .
$$

The first purpose of this paper is to consider results of the above type for solutions to (1.1) satisfying the conditions (1.2) and (1.3).

Theorem 2.1. Suppose that $g \in \mathcal{C}(\overline{\mathbb{D}})$ and $\gamma \in \mathcal{C}(\mathbb{T})$, and that $w \in \mathcal{C}^{4}(\mathbb{D}) \bigcap \mathcal{C}(\overline{\mathbb{D}})$ satisfying the equation (1.1) with the conditions (1.2) and (1.3). Then for $z \in \overline{\mathbb{D}}$, $(2.3)$

$$
\left|w(z)-\frac{1-|z|^{2}}{1+|z|^{2}} \mathcal{P}_{\gamma_{0}}(0)\right| \leq \frac{4}{\pi}\left\|\mathcal{P}_{\gamma_{0}}\right\|_{\infty} \arctan |z|+|c|+\|\gamma\|_{\infty} N_{1}(|z|)+\|g\|_{\infty} N_{2}(|z|),
$$

where

$$
\begin{gathered}
N_{1}(t)=2 \log 4+\frac{1-t^{2}}{2}\left(\frac{2}{3} \pi^{2}-2\right)^{\frac{1}{2}}+4\left(\frac{\pi^{2}}{6}\right)^{\frac{1}{2}} \\
N_{2}(t)=4 \log 4+\left(1-t^{2}\right)\left(\frac{2}{3} \pi^{2}-2\right)^{\frac{1}{2}}+\frac{16}{3}\left(\frac{\pi^{2}}{6}\right)^{\frac{1}{2}}, \\
\left\|\mathcal{P}_{\gamma_{0}}\right\|_{\infty}=\sup _{z \in \mathbb{D}}\left\{\left|\mathcal{P}_{\gamma_{0}}(z)\right|\right\},\|\gamma\|_{\infty}=\sup _{z \in \mathbb{T}}\{|\gamma(z)|\} \text { and }\|g\|_{\infty}=\sup _{z \in \mathbb{D}}\{|g(z)|\} .
\end{gathered}
$$

Clearly, if $c=0, \gamma=g \equiv 0$ and $w$ maps $\mathbb{D}$ into itself, then (2.3) coincides with (2.2).

2.2. A Schwarz-Pick type lemma. For a $2 \times 2$ real matrix $M:=M_{2 \times 2}$, the matrix norm and the matrix function are defined by

$$
\|M\|=\sup \{|M z|: z \in \mathbb{T}\} \text { and } \lambda(M)=\inf \{|M z|: z \in \mathbb{T}\},
$$

respectively. For a complex-valued function $w=f(z)=u(z)+i v(z)$, the Jacobian matrix $D_{f}$ and Jacobian (determinant) $J_{f}$ of $f$ are defined by

$$
D_{f}=\left(\begin{array}{ll}
u_{x} & u_{y} \\
v_{x} & v_{y}
\end{array}\right) \text { and } J_{f}:=\operatorname{det} D_{f}=u_{x} v_{y}-v_{x} u_{y}=\left|f_{z}\right|^{2}-\left|f_{\bar{z}}\right|^{2}
$$

respectively. Obviously

$$
\left\|D_{f}(z)\right\|=\sup \left\{\left|D_{f}(z) \varsigma\right|:|\varsigma|=1\right\}=\left|f_{z}(z)\right|+\left|f_{\bar{z}}(z)\right|
$$

and

$$
\lambda\left(D_{f}(z)\right)=\inf \left\{\left|D_{f}(z) \varsigma\right|:|\varsigma|=1\right\}=|| f_{z}(z)|-| f_{\bar{z}}(z)|| .
$$

Colonna [14] obtained a sharp Schwarz-Pick type lemma for $f \in \mathcal{H}(\mathbb{D}, \mathbb{D})$ :

$$
\left\|D_{f}(z)\right\| \leq \frac{4}{\pi} \frac{1}{1-|z|^{2}} \text { for } z \in \mathbb{D} .
$$

Our second aim in this paper is to prove the following Schwarz-Pick type lemma for solutions to (1.1) satisfying the conditions (1.2) and (1.3). 
Theorem 2.2. Suppose that $g \in \mathcal{C}(\overline{\mathbb{D}})$ and $\gamma \in \mathcal{C}(\mathbb{T})$, and that $w \in \mathcal{C}^{4}(\mathbb{D}) \bigcap \mathcal{C}(\overline{\mathbb{D}})$ satisfying the equation (1.1) with the conditions (1.2) and (1.3). Then for $z \in \mathbb{D}$,

$$
\left\|D_{w}(z)\right\| \leq \frac{4}{\pi}\left\|\mathcal{P}_{\gamma_{0}}\right\|_{\infty} \frac{1}{1-|z|^{2}}+2|c|+\|\gamma\|_{\infty} N_{3}(|z|)+\|g\|_{\infty} N_{4}(|z|),
$$

where

$$
\begin{aligned}
& N_{3}(t)=2\left(\frac{\pi^{2}}{3}+1\right)^{\frac{1}{2}}+t\left(\frac{2}{3} \pi^{2}-2\right)^{\frac{1}{2}}+\left(1-t^{2}\right)\left(\frac{\pi^{2}}{6}-1\right)^{\frac{1}{2}}+\left(\frac{\pi^{2}}{3}-\frac{1}{2}\right)^{\frac{1}{2}} \\
& N_{4}(t)=2(\log 4+1)+t\left(\frac{2}{3} \pi^{2}-2\right)^{\frac{1}{2}}+\frac{2}{3}\left(1-t^{2}\right)\left(\frac{\pi^{2}}{6}-1\right)^{\frac{1}{2}}+\frac{2}{3}\left(\frac{\pi^{2}}{3}-\frac{1}{2}\right)^{\frac{1}{2}}
\end{aligned}
$$

$\left\|\mathcal{P}_{\gamma_{0}}\right\|_{\infty},\|\gamma\|_{\infty}$ and $\|g\|_{\infty}$ are as in Theorem 2.1 .

Remark 2.1. We note that if $c=0, \gamma=g \equiv 0$ and $w$ maps $\mathbb{D}$ into itself, then (2.6) coincides with (2.5).

2.3. A Landau type theorem. The classical Landau theorem says that there is a $\rho=\frac{1}{M+\sqrt{M^{2}-1}}$ such that every function $f$, analytic in $\mathbb{D}$ with $f(0)=f^{\prime}(0)-1=0$ and $|f(z)|<M$, is univalent in the disk $\mathbb{D}_{\rho}$. Moreover, the range $f\left(\mathbb{D}_{\rho}\right)$ contains a disk of radius $M \rho^{2}$, where $M \geq 1$ is a constant (see [18]). The Landau theorem has become an important tool in geometric function theory of one complex variable (cf. [4, 24]). Unfortunately, for a general class of functions, there is no Landau type theorem (cf. [15, 23]). To establish analogs of the Landau type theorem for more general classes of functions, it is necessary to restrict our focus to certain subclasses (cf. [1, 5, 6, 8, 9, 11, 12, 21, 23).

As an application of Theorem 2.2, we get the following Landau type theorem for the solutions to (1.1) with the conditions (1.2) and (1.3).

Theorem 2.3. Suppose that $\gamma \in C(\mathbb{T}), g \in C(\overline{\mathbb{D}})$, that $w \in \mathcal{C}^{4}(\mathbb{D}) \bigcap \mathcal{C}(\overline{\mathbb{D}})$ satisfying the equation (1.1) with the conditions (1.2), (1.3) and $w(0)=J_{w}(0)-1=0$, and that $\left\|\gamma_{0}\right\|_{\infty} \leq L_{1},\|\gamma\|_{\infty} \leq L_{2}$ and $\|g\|_{\infty} \leq L_{3}$, where $L_{j}, j \in\{1,2,3\}$, are constants. Then

(1) $w$ is univalent in $\mathbb{D}_{r_{0}}$, where $r_{0}$ satisfies the following equation

$$
\frac{1}{L_{4}}-2 r_{0}\left(\frac{4 L_{1}}{\pi} \frac{2-r_{0}}{\left(1-r_{0}\right)^{2}}+L_{5}\right)-8 L_{3} \log \frac{1+r_{0}}{1-r_{0}}=0 ;
$$

$(2) w\left(\mathbb{D}_{r_{0}}\right)$ contains a univalent disk $\mathbb{D}_{R_{0}}$ with

$$
R_{0} \geq \frac{8 L_{1}}{\pi}\left(\frac{r_{0}}{1-r_{0}}\right)^{2}+L_{5} r_{0}^{2}+\frac{8 L_{3} r_{0}^{2}\left(3-r_{0}^{2}\right)}{\left(1-r_{0}^{2}\right)^{2}}
$$

where

$$
L_{4}=2|c|+\frac{4}{\pi} L_{1}+L_{2} N_{3}(0)+L_{3} N_{4}(0), \quad L_{5}=|c|+L_{2} M_{1}+L_{3} M_{2},
$$

$N_{3}(0)$ and $N_{4}(0)$ are defined in Theorem 2.2, whereas $M_{1}$ and $M_{2}$ are defined in Lemmas 3.1 and 3.2, respectively. 
We would like to remark that this article continues the earlier study on this topic from [19]. Recently, many authors have studied the Schwarz type lemma, SchwarzPick type lemma and Landau type theorem for solutions of different equations (cf. [7, 8, 13, 20, 21]).

The rest of this article is organized as follows. Section 3 is devoted to stating and proving several useful lemmas. In Section 4 , we present the proofs of Theorems 2.1, 2.2 and 2.3 .

\section{Several Basic Lemmas}

In this section, we shall prove three lemmas which will be used later on.

Lemma 3.1. Suppose $\gamma \in C(\mathbb{T})$, and $\mathcal{G}_{1}[\gamma]$ is defined by (1.5). Then for $z \in \mathbb{D}$,

$$
\left|\frac{\partial}{\partial z} \mathcal{G}_{1}[\gamma](z)-\frac{\partial}{\partial z} \mathcal{G}_{1}[\gamma](0)\right| \leq\left(\|\gamma\|_{\infty} M_{1}\right)|z|
$$

and

$$
\left|\frac{\partial}{\partial \bar{z}} \mathcal{G}_{1}[\gamma](z)-\frac{\partial}{\partial \bar{z}} \mathcal{G}_{1}[\gamma](z)\right| \leq\left(\|\gamma\|_{\infty} M_{1}\right)|z|
$$

where

$$
M_{1}=\frac{1}{2}\left[\frac{2 \pi}{\sqrt{3}}+1+\left(\frac{2}{3} \pi^{2}-2\right)^{\frac{1}{2}}+\left(\frac{\pi^{2}}{6}-\frac{5}{4}\right)^{\frac{1}{2}}+\left(\frac{\pi^{2}}{6}-1\right)^{\frac{1}{2}}+\left(\frac{\pi^{2}}{3}-\frac{11}{4}\right)^{\frac{1}{2}}\right] \text {. }
$$

Proof. To prove the lemma, we only need to show the first inequality, namely,

$$
\left|\frac{\partial}{\partial z} \mathcal{G}_{1}[\gamma](z)-\frac{\partial}{\partial z} \mathcal{G}_{1}[\gamma](0)\right| \leq\left(\|\gamma\|_{\infty} M_{1}\right)|z|
$$

since the proof of the second inequality is similar. Let

$$
\begin{aligned}
& I_{1}(z)=\frac{1}{2 \pi} \int_{0}^{2 \pi}\left(e^{-i t}-\bar{z}\right)\left(\log \left|1-z e^{-i t}\right|^{2}+1\right) \gamma\left(e^{i t}\right) d t \\
& I_{2}(z)=\frac{1}{2 \pi} \int_{0}^{2 \pi} \bar{z}\left[4+\frac{1-z e^{-i t}}{z e^{-i t}} \log \left(1-z e^{-i t}\right)+\frac{1-\bar{z} e^{i t}}{\bar{z} e^{i t}} \log \left(1-\bar{z} e^{i t}\right)\right] \gamma\left(e^{i t}\right) d t \\
& I_{3}(z)=\frac{1}{2 \pi} \int_{0}^{2 \pi}\left(1-|z|^{2}\right)\left(\frac{\log \left(1-z e^{-i t}\right)}{z^{2} e^{-i t}}+\frac{1}{z}\right) \gamma\left(e^{i t}\right) d t, \text { and } \\
& I_{4}(z)=\frac{1}{2 \pi} \int_{0}^{2 \pi}\left(\frac{e^{i t}-z^{2} e^{-i t}}{z^{2}} \log \left(1-z e^{-i t}\right)+\frac{1-z e^{-i t}}{z}\right) \gamma\left(e^{i t}\right) d t .
\end{aligned}
$$

Now, we need to estimate $\left|I_{j}(z)-I_{j}(0)\right|$ for $j=1,2,3,4$, respectively.

Claim 3.1. $\left|I_{1}(z)-I_{1}(0)\right| \leq\|\gamma\|_{\infty}|z|\left[2\left(\frac{\pi^{2}}{3}\right)^{\frac{1}{2}}+1\right]$.

By elementary calculations, we get

$$
\left|I_{1}(z)-I_{1}(0)\right| \leq\|\gamma\|_{\infty}\left(2 J_{1}(z)+|z|\right),
$$


where

$$
J_{1}(z)=\frac{1}{2 \pi} \int_{0}^{2 \pi}|\log | 1-\left.z e^{-i t}\right|^{2}\left|d t=\frac{1}{2 \pi} \int_{0}^{2 \pi}\right| \sum_{n=1}^{\infty} \frac{z^{n} e^{-i n t}}{n}+\sum_{n=1}^{\infty} \frac{\bar{z}^{n} e^{i n t}}{n} \mid d t .
$$

By the Hölder inequality and Parseval's theorem, we obtain

$$
\begin{aligned}
J_{1}(z) & \leq\left(\frac{1}{2 \pi} \int_{0}^{2 \pi}\left|\sum_{n=1}^{\infty} \frac{z^{n} e^{-i n t}}{n}+\sum_{n=1}^{\infty} \frac{\bar{z}^{n} e^{i n t}}{n}\right|^{2} d t\right)^{\frac{1}{2}} \\
& =\left(2 \sum_{n=1}^{\infty} \frac{|z|^{2 n}}{n^{2}}\right)^{\frac{1}{2}} \leq|z|\left(\frac{\pi^{2}}{3}\right)^{\frac{1}{2}}
\end{aligned}
$$

which proves Claim 3.1 .

Claim 3.2. $\left|I_{2}(z)-I_{2}(0)\right| \leq\|\gamma\|_{\infty}|z|\left(\frac{2}{3} \pi^{2}-2\right)^{\frac{1}{2}}$.

Since $\left|I_{2}(z)-I_{2}(0)\right|=\left|I_{2}(z)\right|$, Claim 3.2 follows from [19, Claim 2.4].

Claim 3.3. $\left|I_{3}(z)-I_{3}(0)\right| \leq\|\gamma\|_{\infty}|z|\left[\left(\frac{\pi^{2}}{6}-\frac{5}{4}\right)^{\frac{1}{2}}+\left(\frac{\pi^{2}}{6}-1\right)^{\frac{1}{2}}\right]$.

Since

$$
\frac{\log \left(1-z e^{-i t}\right)}{z^{2} e^{-i t}}+\frac{1}{z}=-\sum_{n=1}^{\infty} \frac{z^{n-1} e^{-i n t}}{n+1}
$$

we deduce that

$$
I_{3}(0)=\frac{1}{2 \pi} \int_{0}^{2 \pi}\left(-\frac{e^{-i t}}{2}\right) \gamma\left(e^{i t}\right) d t
$$

Then

$$
\left|I_{3}(z)-I_{3}(0)\right| \leq\|\gamma\|_{\infty}\left(J_{2}(z)+J_{3}(z)\right)
$$

where

$$
J_{2}(z)=\frac{1}{2 \pi} \int_{0}^{2 \pi}\left|\sum_{n=2}^{\infty} \frac{z^{n-1} e^{-i n t}}{n+1}\right| d t \text { and } J_{3}(z)=\frac{|z|^{2}}{2 \pi} \int_{0}^{2 \pi}\left|\sum_{n=1}^{\infty} \frac{z^{n-1} e^{-i n t}}{n+1}\right| d t .
$$

As in the proof of the estimate for $J_{1}(z)$, by the Hölder inequality and Parseval's theorem, we obtain

$$
\begin{aligned}
J_{2}(z) & \leq\left(\frac{1}{2 \pi} \int_{0}^{2 \pi}\left|\sum_{n=2}^{\infty} \frac{z^{n-1} e^{-i n t}}{n+1}\right|^{2} d t\right)^{\frac{1}{2}} \\
& =\left(\sum_{n=2}^{\infty} \frac{|z|^{2(n-1)}}{(n+1)^{2}}\right)^{\frac{1}{2}} \leq|z|\left(\frac{\pi^{2}}{6}-\frac{5}{4}\right)^{\frac{1}{2}} .
\end{aligned}
$$


Similarly, we know that

$$
J_{3}(z) \leq|z|^{2}\left(\frac{\pi^{2}}{6}-1\right)^{\frac{1}{2}} .
$$

Claim 3.3 follows as $|z|<1$.

Claim 3.4. $\left|I_{4}(z)-I_{4}(0)\right| \leq\|\gamma\|_{\infty}|z|\left(\frac{\pi^{2}}{3}-\frac{11}{4}\right)^{\frac{1}{2}}$.

We rewrite $I_{4}(z)$ as

$$
I_{4}(z)=\frac{1}{2 \pi} \int_{0}^{2 \pi} e^{-i t} G\left(z e^{-i t}\right) \gamma\left(e^{i t}\right) d t
$$

where

$$
G(z)=\frac{1-z^{2}}{z^{2}} \log (1-z)+\frac{1}{z}-1=2 \sum_{n=1}^{\infty} \frac{z^{n}}{n(n+2)}-\frac{3}{2} .
$$

Then $I_{4}(0)=\frac{1}{2 \pi} \int_{0}^{2 \pi}\left(-\frac{3}{2}\right) e^{-i t} \gamma\left(e^{i t}\right) d t$. As before, we find that

$$
\begin{aligned}
\left|I_{4}(z)-I_{4}(0)\right| & \leq \frac{\|\gamma\|_{\infty}}{2 \pi} \int_{0}^{2 \pi}\left|2 \sum_{n=1}^{\infty} \frac{z^{n} e^{-i n t}}{n(n+2)}\right| d t \\
& \leq\|\gamma\|_{\infty}\left(\frac{1}{2 \pi} \int_{0}^{2 \pi}\left|2 \sum_{n=1}^{\infty} \frac{z^{n} e^{-i n t}}{n(n+2)}\right|^{2} d t\right)^{\frac{1}{2}} \\
& =\|\gamma\|_{\infty}\left(\sum_{n=1}^{\infty} \frac{4|z|^{2 n}}{n^{2}(n+2)^{2}}\right)^{\frac{1}{2}} \leq\|\gamma\|_{\infty}|z|\left(\frac{\pi^{2}}{3}-\frac{11}{4}\right)^{\frac{1}{2}},
\end{aligned}
$$

since

$$
\sum_{n=1}^{\infty} \frac{4}{n^{2}(n+2)^{2}}=\sum_{n=1}^{\infty} \frac{1}{(n+2)^{2}}+\sum_{n=1}^{\infty} \frac{1}{n^{2}}-2 \sum_{n=1}^{\infty} \frac{1}{n(n+2)}=\frac{\pi^{2}}{3}-\frac{11}{4},
$$

and Claim 3.4 follows.

Therefore, by Claims [3.1, 3.2, 3.3, 3.4 and [17, Proposition 2.4], we conclude that

$$
\left|\frac{\partial}{\partial z} \mathcal{G}_{1}[\gamma](z)-\frac{\partial}{\partial z} \mathcal{G}_{1}[\gamma](0)\right| \leq \frac{1}{2} \sum_{j=1}^{4}\left|I_{j}(z)-I_{j}(0)\right| \leq\left(\|\gamma\|_{\infty} M_{1}\right)|z|,
$$

as required.

Lemma 3.2. Suppose $g \in C(\overline{\mathbb{D}})$ and $\mathcal{G}_{2}[g]$ is defined in (1.6). Then for $z \in \mathbb{D}_{r^{\star}}$,

$$
\left|\frac{\partial}{\partial z} \mathcal{G}_{2}[g](z)-\frac{\partial}{\partial z} \mathcal{G}_{2}[g](0)\right| \leq\|g\|_{\infty}|z| M_{2}+4\|g\|_{\infty} \log \frac{1+|z|}{1-|z|}
$$

and

$$
\left|\frac{\partial}{\partial \bar{z}} \mathcal{G}_{2}[g](z)-\frac{\partial}{\partial \bar{z}} \mathcal{G}_{2}[g](0)\right| \leq\|g\|_{\infty}|z| M_{2}+4\|g\|_{\infty} \log \frac{1+|z|}{1-|z|}
$$


where $0 \leq r^{\star}<2 r_{0}$ and $r_{0}$ is determined by Eqn. (2.7), and

$M_{2}=\log 4+1+\left(\frac{2}{3} \pi^{2}-2\right)^{\frac{1}{2}}+\frac{2}{3}\left[\left(\frac{\pi^{2}}{6}-\frac{5}{4}\right)^{\frac{1}{2}}+\left(\frac{\pi^{2}}{6}-1\right)^{\frac{1}{2}}+\left(\frac{\pi^{2}}{3}-\frac{11}{4}\right)^{\frac{1}{2}}\right]$.

Proof. To prove the two inequalities, we only need to prove the first inequality, namely,

$$
\left|\frac{\partial}{\partial z} \mathcal{G}_{2}[g](z)-\frac{\partial}{\partial z} \mathcal{G}_{2}[g](0)\right| \leq\left(\|g\|_{\infty} M_{2}\right)|z|
$$

because the proof of the second inequality is similar. To do this, we let

$$
\begin{aligned}
& I_{5}(z)=\int_{\mathbb{D}}(\bar{\zeta}-\bar{z})\left(\log |\zeta-z|^{2}+1\right) g(\zeta) d A(\zeta), \\
& I_{6}(z)=\int_{\mathbb{D}} \bar{z}\left[4+\frac{1-z \bar{\zeta}}{z \bar{\zeta}} \log (1-z \bar{\zeta})+\frac{1-\bar{z} \zeta}{\bar{z} \zeta} \log (1-\bar{z} \zeta)\right] g(\zeta) d A(\zeta), \\
& I_{7}(z)=\int_{\mathbb{D}}\left(1-|z|^{2}\right)\left(\frac{\log (1-z \bar{\zeta})}{z^{2} \bar{\zeta}}+\frac{1}{z}\right) g(\zeta) d A(\zeta), \text { and } \\
& I_{8}(z)=\int_{\mathbb{D}}\left(\frac{\zeta-z^{2} \bar{\zeta}}{z^{2}} \log (1-z \bar{\zeta})+\frac{|\zeta|^{2}-z \bar{\zeta}}{z}\right) g(\zeta) d A(\zeta) .
\end{aligned}
$$

In the following, we estimate $\left|I_{j}(z)-I_{j}(0)\right|$ for $j=5,6,7,8$, respectively.

Claim 3.5. $\left|I_{5}(z)-I_{5}(0)\right| \leq\|g\|_{\infty}\left[4 \log \frac{1+|z|}{1-|z|}+(\log 4+1)|z|\right]$.

By calculations, we get

$$
I_{5}(z)-I_{5}(0)=-J_{4}(z)+J_{5}(z)
$$

where

$$
J_{4}(z)=\int_{\mathbb{D}} \bar{z}\left(\log |\zeta-z|^{2}+1\right) g(\zeta) d A(\zeta)
$$

and

$$
J_{5}(z)=\int_{\mathbb{D}} \bar{\zeta}\left(\log |\zeta-z|^{2}-\log |\zeta|^{2}\right) g(\zeta) d A(\zeta)
$$

Obviously,

$$
\left|J_{4}(z)\right| \leq(\log 4+1)\|g\|_{\infty}|z| .
$$

In order to estimate $J_{5}(z)$, we let

$$
h(z, \zeta)=\bar{\zeta} \log |\zeta-z|^{2} .
$$


Then, for $z \in \mathbb{D}_{r^{\star}}$, by Fubini's Theorem, we get

$$
\begin{aligned}
\left|J_{5}(z)\right| & \leq\|g\|_{\infty} \int_{\mathbb{D}}|h(z, \zeta)-h(0, \zeta)| d A(\zeta) \\
& \leq\|g\|_{\infty} \int_{\mathbb{D}}\left(\int_{[0, z]}\left|h_{z}(z, \zeta) d z+h_{\bar{z}}(z, \zeta) d \bar{z}\right|\right) d A(\zeta) \\
& \leq\|g\|_{\infty} \int_{\mathbb{D}}\left(\int_{[0, z]}\left(\left|h_{z}(z, \zeta)\right|+\left|h_{\bar{z}}(z, \zeta)\right|\right)|d z|\right) d A(\zeta) \\
& =\|g\|_{\infty} \int_{[0, z]} H(z, \zeta)|d z|
\end{aligned}
$$

where

$$
H(z, \zeta)=\int_{\mathbb{D}}\left(\left|h_{z}(z, \zeta)\right|+\left|h_{\bar{z}}(z, \zeta)\right|\right) d A(\zeta)=2 \int_{\mathbb{D}}\left|\frac{\zeta}{\zeta-z}\right| d A(\zeta) .
$$

In order to estimate $H(z, \zeta)$, we let

$$
\zeta \mapsto \eta=\phi(\zeta)=\frac{z-\zeta}{1-\zeta \bar{z}}=r e^{i \theta}
$$

so that $\phi=\phi^{-1}$,

$$
\zeta=\frac{z-\eta}{1-\eta \bar{z}}, z-\zeta=\frac{\eta\left(1-|z|^{2}\right)}{1-\eta \bar{z}}, \phi^{\prime}(\zeta)=-\frac{1-|z|^{2}}{(1-\zeta \bar{z})^{2}}
$$

and thus,

$$
d A(\zeta)=\left|\left(\phi^{-1}\right)^{\prime}(\eta)\right|^{2} d A(\eta)=\frac{\left(1-|z|^{2}\right)^{2}}{|1-\eta \bar{z}|^{4}} d A(\eta) .
$$

Consequently, switching to polar coordinates yields

$$
H(z, \zeta)=\int_{\mathbb{D}} \frac{|z-\eta|\left(1-|z|^{2}\right)}{|\eta| \cdot|1-\eta \bar{z}|^{4}} d A(\eta) \leq \frac{2\left(1-|z|^{2}\right)}{\pi} \int_{0}^{1} \int_{0}^{2 \pi} \frac{1}{\left|1-\bar{z} r e^{i \theta}\right|^{4}} d \theta d r .
$$

By Parseval's theorem, we get

$$
\frac{1}{2 \pi} \int_{0}^{2 \pi} \frac{d \theta}{\left|1-\bar{z} r e^{-i \theta}\right|^{4}}=\sum_{n=0}^{\infty}(n+1)^{2}|z|^{2 n} r^{2 n}
$$

and thus,

$$
H(z, \zeta) \leq 4\left(1-|z|^{2}\right) \sum_{n=0}^{\infty} \frac{(n+1)^{2}}{2 n+1}|z|^{2 n} \leq 4\left(1-|z|^{2}\right) \sum_{n=0}^{\infty}(n+1)|z|^{2 n}=\frac{4}{1-|z|^{2}}
$$

because $\sum_{n=0}^{\infty}(n+1) z^{n}=1 /(1-z)^{2}$ for all $|z|<1$. Hence,

$$
\left|J_{5}(z)\right| \leq\|g\|_{\infty} \int_{[0, z]} \frac{8}{1-|z|^{2}}|d z|=4\|g\|_{\infty} \log \frac{1+|z|}{1-|z|} .
$$

Claim 3.5 follows from $\left|I_{5}(z)-I_{5}(0)\right| \leq\left|J_{4}(z)\right|+\left|J_{5}(z)\right|$, (3.1) and (3.2).

In the following, we let $\zeta=\rho e^{i t}$. By calculations and the Hölder inequality, we get 
Claim 3.6. $\left|I_{6}(z)-I_{6}(0)\right| \leq\|g\|_{\infty}|z|\left(\frac{2}{3} \pi^{2}-2\right)^{\frac{1}{2}}$.

Claim 3.6 follows from [19, Claim 2.8], because $\left|I_{6}(z)-I_{6}(0)\right|=\left|I_{6}(z)\right|$.

Claim 3.7. $\left|I_{7}(z)-I_{7}(0)\right| \leq \frac{2}{3}\|g\|_{\infty}|z|\left[\left(\frac{\pi^{2}}{6}-\frac{5}{4}\right)^{\frac{1}{2}}+\left(\frac{\pi^{2}}{6}-1\right)^{\frac{1}{2}}\right]$.

As in the proof of Claim 3.3 in Lemma 3.1, we use the representation

$$
\frac{\log (1-z \bar{\zeta})}{z^{2} \bar{\zeta}}+\frac{1}{z}=-\bar{\zeta} \sum_{n=1}^{\infty} \frac{(z \bar{\zeta})^{n-1}}{n+1}
$$

and obtain that

where

$$
\left|I_{7}(z)-I_{7}(0)\right| \leq\|g\|_{\infty}\left(J_{6}(z)+J_{7}(z)\right)
$$

$$
J_{6}(z)=\int_{\mathbb{D}}\left|\sum_{n=2}^{\infty} \frac{(z \bar{\zeta})^{n-1}}{n+1}\right||\zeta| d A(\zeta) \text { and } J_{7}(z)=|z|^{2} \int_{\mathbb{D}}\left|\sum_{n=1}^{\infty} \frac{(z \bar{\zeta})^{n-1}}{n+1}\right||\zeta| d A(\zeta) .
$$

By Hölder's inequality and Parseval's theorem, we get

$$
\begin{aligned}
J_{6}(z) & \leq 2 \int_{0}^{1}\left(\frac{1}{2 \pi} \int_{0}^{2 \pi}\left|\sum_{n=2}^{\infty} \frac{\left(z \rho e^{-i t}\right)^{n-1}}{n+1}\right|^{2} d t\right)^{\frac{1}{2}} \rho^{2} d \rho \\
& =2 \int_{0}^{1}\left(\sum_{n=1}^{\infty} \frac{|z|^{2 n} \rho^{2 n}}{(n+2)^{2}}\right)^{\frac{1}{2}} \rho^{2} d \rho \\
& \leq 2|z|\left(\sum_{n=1}^{\infty} \frac{1}{(n+2)^{2}}\right)^{\frac{1}{2}} \int_{0}^{1} \rho^{2} d \rho=\frac{2}{3}|z|\left(\frac{\pi^{2}}{6}-\frac{5}{4}\right)^{\frac{1}{2}} .
\end{aligned}
$$

By similar reasoning as above, one obtains that

$$
J_{7}(z) \leq \frac{2}{3}|z|\left(\frac{\pi^{2}}{6}-1\right)^{\frac{1}{2}}
$$

The Claim 3.7 follows.

Claim 3.8. $\left|I_{8}(z)-I_{8}(0)\right| \leq \frac{2}{3}\|g\|_{\infty}|z|\left(\frac{\pi^{2}}{3}-\frac{11}{4}\right)^{\frac{1}{2}}$.

It follows from

$$
\frac{\zeta-z^{2} \bar{\zeta}}{z^{2}} \log (1-z \bar{\zeta})+\frac{|\zeta|^{2}-z \bar{\zeta}}{z}=\bar{\zeta}\left(\sum_{n=1}^{\infty} \frac{n\left(1-|\zeta|^{2}\right)+2}{n(n+2)}(z \bar{\zeta})^{n}-1-\frac{|\zeta|^{2}}{2}\right)
$$

that

$$
\left|I_{8}(z)-I_{8}(0)\right|=\left|\int_{\mathbb{D}} \bar{\zeta} \sum_{n=1}^{\infty} \frac{n\left(1-|\zeta|^{2}\right)+2}{n(n+2)}(z \bar{\zeta})^{n} g(\zeta) d A(\zeta)\right| \leq\|g\|_{\infty} J_{8}(z),
$$


where

$$
J_{8}(z)=\int_{\mathbb{D}}\left|\sum_{n=1}^{\infty} \frac{n\left(1-|\zeta|^{2}\right)+2}{n(n+2)}(z \bar{\zeta})^{n}\right| \cdot|\zeta| d A(\zeta) .
$$

Now it is easy to see that

$$
J_{8}(z) \leq 2 \int_{0}^{1} \Psi(z, \rho) \rho^{2} d \rho, \quad \zeta=\rho e^{i t}
$$

where

$$
\begin{aligned}
\Psi(z, \rho) & =\left(\frac{1}{2 \pi} \int_{0}^{2 \pi}\left|\sum_{n=1}^{\infty} \frac{n\left(1-\rho^{2}\right)+2}{n(n+2)}\left(z \rho e^{-i t}\right)^{n}\right|^{2} d t\right)^{\frac{1}{2}} \\
& =\left(\sum_{n=1}^{\infty} \frac{\left(n\left(1-\rho^{2}\right)+2\right)^{2} \rho^{2 n}}{n^{2}(n+2)^{2}}|z|^{2 n}\right)^{\frac{1}{2}} \\
& \leq|z|\left(4 \sum_{n=1}^{\infty} \frac{1}{n^{2}(n+2)^{2}}\right)^{\frac{1}{2}}=|z|\left(\frac{\pi^{2}}{3}-\frac{11}{4}\right)^{\frac{1}{2}} .
\end{aligned}
$$

Here we have used the fact that for each $n \geq 1, \varphi(\rho)=\left(n\left(1-\rho^{2}\right)+2\right)^{2} \rho^{2 n}$ is an increasing function $\rho$. The Claim 3.8 follows.

Now, Claims [3.5, 3.6, 3.7, 3.8 and [17, Proposition 2.4] guarantee that

$$
\left|\frac{\partial}{\partial z} \mathcal{G}_{2}[g](z)-\frac{\partial}{\partial z} \mathcal{G}_{2}[g](0)\right| \leq \sum_{j=5}^{8}\left|I_{j}(z)-I_{j}(0)\right| \leq\|g\|_{\infty}|z| M_{2}+4\|g\|_{\infty} \log \frac{1+|z|}{1-|z|}
$$

as required.

Lemma 3.3. For constants $C_{j}>0, j \in\{1,2,3,4\}$, let

$$
\varphi(x)=C_{1}-C_{2} x\left[\frac{2-x}{(1-x)^{2}}+C_{3}\right]-C_{4} \log \frac{1+x}{1-x}, \quad x \in[0,1) .
$$

Then we have

(1) $\varphi$ is continuous and strictly decreasing in $(0,1)$;

(2) there is a unique $x_{0} \in(0,1)$ such that $\varphi\left(x_{0}\right)=0$.

Proof. For $x \in[0,1)$, we find that

$$
\varphi^{\prime}(x)=-\frac{2 C_{2}}{(1-x)^{3}}-C_{2} C_{3}-\frac{2 C_{4}}{1-x^{2}}<0
$$

showing that $\varphi(x)$ is strictly decreasing in $[0,1)$. Moreover,

$$
\varphi(0)=C_{1}>0 \text { and } \lim _{x \rightarrow 1^{-}} \varphi(x)=-\infty<0
$$

which implies that there is a unique $x_{0} \in(0,1)$ such that $\varphi\left(x_{0}\right)=0$. The proof of the lemma is complete. 


\section{The Proof of main Results}

In this section, we supply the proofs of Theorems 2.1, 2.2 and 2.3.

Proof of Theorem 2.1. By (1.4) and (2.2), we can quickly deduce that

$$
\left|w(z)-\frac{1-|z|^{2}}{1+|z|^{2}} \mathcal{P}_{\gamma_{0}}(0)\right| \leq \frac{4}{\pi}\left\|\mathcal{P}_{\gamma_{0}}\right\|_{\infty} \arctan |z|+|c|+\left|\mathcal{G}_{1}[\gamma](z)\right|+\left|\mathcal{G}_{2}[g](z)\right| .
$$

We just need to estimate $\left|\mathcal{G}_{1}[\gamma](z)\right|$ and $\left|\mathcal{G}_{2}[g](z)\right|$.

Claim 4.1. $\left|\mathcal{G}_{1}[\gamma](z)\right| \leq\|\gamma\|_{\infty} N_{1}(|z|)$.

By elementary calculations, (1.5) and Claim 3.2, we obtain

$$
\left|\mathcal{G}_{1}[\gamma](z)\right| \leq \frac{\|\gamma\|_{\infty}}{2}\left[4 \log 4+\left(1-|z|^{2}\right)\left(\frac{2}{3} \pi^{2}-2\right)^{\frac{1}{2}}\right]+4\|\gamma\|_{\infty} J_{9}(z),
$$

where

$$
J_{9}(z)=\frac{1}{2 \pi} \int_{0}^{2 \pi}\left|\frac{\log \left(1-z e^{-i t}\right)}{z}\right| d t .
$$

Now, in order to estimate $J_{9}(z)$, we use Hölder's inequality and Parseval's theorem to get

$$
J_{9}(z) \leq\left(\frac{1}{2 \pi} \int_{0}^{2 \pi}\left|\sum_{n=1}^{\infty} \frac{z^{n-1} e^{-i n t}}{n}\right|^{2} d t\right)^{\frac{1}{2}}=\left(\sum_{n=1}^{\infty} \frac{|z|^{2(n-1)}}{n^{2}}\right)^{\frac{1}{2}} \leq\left(\frac{\pi^{2}}{6}\right)^{\frac{1}{2}}
$$

which proves Claim 4.1,

Claim 4.2. $\left|\mathcal{G}_{2}[g](z)\right| \leq \|\left. g\right|_{\infty} N_{2}(|z|)$.

By elementary calculations, (1.6) and Claim 3.6, we obtain

$$
\left|\mathcal{G}_{2}[g](z)\right| \leq\|g\|_{\infty}\left[4 \log 4+\left(1-|z|^{2}\right)\left(\frac{2}{3} \pi^{2}-2\right)^{\frac{1}{2}}\right]+8\|g\|_{\infty} J_{10}(z),
$$

where

$$
J_{10}(z)=\int_{\mathbb{D}}\left|\frac{\log (1-z \bar{\zeta})}{z}\right| d A(\zeta)
$$

In order to estimate $J_{10}(z)$, we let $\zeta=\rho e^{i t}$. Switching to polar coordinates and by Hölder's inequality and Parseval's theorem, we get

$$
\begin{aligned}
J_{10}(z) & \leq 2 \int_{0}^{1}\left(\frac{1}{2 \pi} \int_{0}^{2 \pi}\left|\sum_{n=1}^{\infty} \frac{\left(z \rho e^{-i t}\right)^{n-1}}{n}\right|^{2} d t\right)^{\frac{1}{2}} \rho^{2} d \rho \\
& \leq 2\left(\frac{\pi^{2}}{6}\right)^{\frac{1}{2}} \int_{0}^{1} \rho^{2} d \rho=\frac{2}{3}\left(\frac{\pi^{2}}{6}\right)^{\frac{1}{2}}
\end{aligned}
$$

which proves Claim 4.2 ,

Hence, it follows from Claim 4.1 and Claim 4.2 that (2.3) holds, and the proof of the theorem is complete. 
Proof of Theorem 2.2. By (1.4) and (2.4), for each $z \in \mathbb{D}$, we get

$$
\left\|D_{w}(z)\right\|=\left|w_{z}(z)\right|+\left|w_{\bar{z}}(z)\right| \leq 2|c|+\left\|D_{\mathcal{P}_{\gamma_{0}}}(z)\right\|+\left\|D_{\mathcal{G}_{1}[\gamma]}(z)\right\|+\left\|D_{\mathcal{G}_{2}[g]}(z)\right\| .
$$

From (2.5) and [19, Lemmas 2.2 and 2.3], we deduce that

$$
\left\|D_{w}(z)\right\| \leq \frac{4}{\pi}\left\|\mathcal{P}_{\gamma_{0}}\right\|_{\infty} \frac{1}{1-|z|^{2}}+2|c|+\|\gamma\|_{\infty} N_{3}(|z|)+\|g\|_{\infty} N_{4}(|z|)
$$

as required, and the proof of the theorem is complete.

Before we prove Theorem [2.3, let us recall the following result.

Theorem A. 11, Lemma 1] Suppose $f$ is a harmonic mapping of $\mathbb{D}$ into $\mathbb{C}$ such that $|f(z)| \leq M$ and $f(z)=\sum_{n=0}^{\infty} a_{n} z^{n}+\sum_{n=1}^{\infty} \bar{b}_{n} \bar{z}^{n}$. Then $\left|a_{0}\right| \leq M$ and for all $n \geq 1$,

$$
\left|a_{n}\right|+\left|b_{n}\right| \leq \frac{4 M}{\pi}
$$

This estimate is sharp, and the extreme function is

$$
f_{n}(z)= \begin{cases}\frac{2 M \alpha_{1}}{\pi} \arg \left(\frac{1+\beta_{1} z^{n}}{1-\beta_{1} z^{n}}\right),\left|\alpha_{1}\right|=\left|\beta_{1}\right|=1, & \text { if } n \geq 1 \\ M & \text { if } n=0 .\end{cases}
$$

Proof of Theorem 2.3. The function $\mathcal{P}_{\gamma_{0}}$ is harmonic in $\mathbb{D}$ and thus, it can be written in the form

$$
\mathcal{P}_{\gamma_{0}}(z)=\sum_{n=0}^{\infty} a_{n} z^{n}+\sum_{n=1}^{\infty} \bar{b}_{n} \bar{z}^{n}
$$

Applying Theorem 1, we get

$$
\begin{aligned}
& \left|\frac{\partial}{\partial z} \mathcal{P}_{\gamma_{0}}(z)-\frac{\partial}{\partial z} \mathcal{P}_{\gamma_{0}}(0)\right|+\left|\frac{\partial}{\partial \bar{z}} \mathcal{P}_{\gamma_{0}}(z)-\frac{\partial}{\partial \bar{z}} \mathcal{P}_{\gamma_{0}}(0)\right| \\
& =\left|\sum_{n=2}^{\infty} n a_{n} z^{n-1}\right|+\left|\sum_{n=2}^{\infty} n b_{n} \bar{z}^{n-1}\right| \\
& \quad \leq \sum_{n=2}^{\infty} n\left(\left|a_{n}\right|+\left|b_{n}\right|\right)|z|^{n-1} \leq \frac{4 L_{1}}{\pi} \sum_{n=2}^{\infty} n|z|^{n-1}=\frac{4 L_{1}}{\pi} \frac{|z|(2-|z|)}{(1-|z|)^{2}} .
\end{aligned}
$$

Then by Lemmas 3.1 and 3.2 , and (4.1), for $z \in \mathbb{D}_{r^{\star}}$, we obtain

$$
\begin{aligned}
\left|w_{z}(z)-w_{z}(0)\right| \leq & |c||z|+\left|\frac{\partial}{\partial z} \mathcal{P}_{\gamma_{0}}(z)-\frac{\partial}{\partial z} \mathcal{P}_{\gamma_{0}}(0)\right|+\left|\frac{\partial}{\partial z} \mathcal{G}_{1}[\gamma](z)-\frac{\partial}{\partial z} \mathcal{G}_{1}[\gamma](0)\right| \\
& +\left|\frac{\partial}{\partial z} \mathcal{G}_{2}[g](z)-\frac{\partial}{\partial z} \mathcal{G}_{2}[g](0)\right| \\
\leq & |z|\left(\frac{4 L_{1}}{\pi} \frac{2-|z|}{(1-|z|)^{2}}+L_{5}\right)+4 L_{3} \log \frac{1+|z|}{1-|z|},
\end{aligned}
$$


and

$$
\begin{aligned}
\left|w_{\bar{z}}(z)-w_{\bar{z}}(0)\right| \leq & |c||z|+\left|\frac{\partial}{\partial \bar{z}} \mathcal{P}_{\gamma_{0}}(z)-\frac{\partial}{\partial \bar{z}} \mathcal{P}_{\gamma_{0}}(0)\right|+\left|\frac{\partial}{\partial \bar{z}} \mathcal{G}_{1}[\gamma](z)-\frac{\partial}{\partial \bar{z}} \mathcal{G}_{1}[\gamma](0)\right| \\
& +\left|\frac{\partial}{\partial \bar{z}} \mathcal{G}_{2}[g](z)-\frac{\partial}{\partial \bar{z}} \mathcal{G}_{2}[g](0)\right| \\
(4.3) \leq & |z|\left(\frac{4 L_{1}}{\pi} \frac{2-|z|}{(1-|z|)^{2}}+L_{5}\right)+4 L_{3} \log \frac{1+|z|}{1-|z|}
\end{aligned}
$$

where $L_{5}=|c|+L_{2} M_{1}+L_{3} M_{2}$.

It follows from Theorem 2.2 that

$$
1=J_{w}(0)=\left\|D_{w}(0)\right\| \lambda\left(D_{w}(0)\right) \leq \lambda\left(D_{w}(0)\right) L_{4},
$$

which gives

$$
\lambda\left(D_{w}(0)\right) \geq \frac{1}{L_{4}}
$$

Now, we are ready to finish the proof of the theorem. First, we demonstrate the univalence of the function $w$ in $\mathbb{D}_{r_{0}}$, where $r_{0}$ is determined by Eqn. (2.7). For this, let $z_{1}, z_{2}$ be two points in $\mathbb{D}_{r_{0}}$ with $z_{1} \neq z_{2}$, and denote the segment from $z_{1}$ to $z_{2}$ with the endpoints $z_{1}$ and $z_{2}$ by $\left[z_{1}, z_{2}\right]$. Since

$$
\begin{aligned}
\left|w\left(z_{2}\right)-w\left(z_{1}\right)\right| & =\left|\int_{\left[z_{1}, z_{2}\right]} w_{z}(z) d z+w_{\bar{z}}(z) d \bar{z}\right| \\
\geq & \left|\int_{\left[z_{1}, z_{2}\right]} w_{z}(0) d z+w_{\bar{z}}(0) d \bar{z}\right| \\
& -\left|\int_{\left[z_{1}, z_{2}\right]}\left[w_{z}(z)-w_{z}(0)\right] d z+\left[w_{\bar{z}}(z)-w_{\bar{z}}(0)\right] d \bar{z}\right|,
\end{aligned}
$$

we see from (4.2), (4.3), (4.4) and Lemma 3.3 that

$$
\begin{aligned}
\left|w\left(z_{2}\right)-w\left(z_{1}\right)\right| \geq & \lambda\left(D_{w}(0)\right) \cdot\left|z_{2}-z_{1}\right| \\
& -\left|\int_{\left[z_{1}, z_{2}\right]}\left[w_{z}(z)-w_{z}(0)\right] d z+\left[w_{\bar{z}}(z)-w_{\bar{z}}(0)\right] d \bar{z}\right| \\
> & {\left[\frac{1}{L_{4}}-2 r_{0}\left(\frac{4 L_{1}}{\pi} \frac{2-r_{0}}{\left(1-r_{0}\right)^{2}}+L_{5}\right)-8 L_{3} \log \frac{1+r_{0}}{1-r_{0}}\right]\left|z_{2}-z_{1}\right| } \\
= & 0 .
\end{aligned}
$$

which implies the univalence of $w$ in $\mathbb{D}_{r_{0}}$.

Next, we prove Theorem 2.3(2). For any $\zeta=r_{0} e^{i \theta} \in \partial \mathbb{D}_{r_{0}}$, we obtain that 


$$
\begin{aligned}
|w(\zeta)-w(0)|= & \left|\int_{[0, \zeta]} w_{z}(z) d z+w_{\bar{z}}(z) d \bar{z}\right| \\
\geq & \left|\int_{[0, \zeta]} w_{z}(0) d z+w_{\bar{z}}(0) d \bar{z}\right| \\
& -\left|\int_{[0, \zeta]}\left[w_{z}(z)-w_{z}(0)\right] d z+\left[w_{\bar{z}}(z)-w_{\bar{z}}(0)\right] d \bar{z}\right| \\
\geq & \lambda\left(D_{w}(0)\right) r_{0}-\frac{8 L_{1}}{\pi} \int_{0}^{r_{0}} \frac{|z|(2-|z|)}{(1-|z|)^{2}}|d z|-2 L_{5} \int_{0}^{r_{0}}|z||d z| \\
& -8 L_{3} \int_{0}^{r_{0}} \log \frac{1+|z|}{1-|z|}|d z| \quad\left(\text { by }(4.2) \text { and }\left(\frac{4.3}{1}\right)\right) \\
\geq & \frac{r_{0}}{L_{4}}-\frac{8 L_{1}}{\pi} \frac{r_{0}^{2}}{1-r_{0}}-L_{5} r_{0}^{2}-8 L_{3} r_{0} \log \frac{1+r_{0}}{1-r_{0}}+\frac{8 L_{3} r_{0}^{2}\left(3-r_{0}^{2}\right)}{\left(1-r_{0}^{2}\right)^{2}} \\
= & \frac{8 L_{1}}{\pi}\left(\frac{r_{0}}{1-r_{0}}\right)^{2}+L_{5} r_{0}^{2}+\frac{8 L_{3} r_{0}^{2}\left(3-r_{0}^{2}\right)}{\left(1-r_{0}^{2}\right)^{2}} \quad(\text { by (2.7) }) .
\end{aligned}
$$

Hence $f\left(\mathbb{D}_{r_{0}}\right)$ contains a univalent disk $\mathbb{D}_{R_{0}}$, where

$$
R_{0} \geq \frac{8 L_{1}}{\pi}\left(\frac{r_{0}}{1-r_{0}}\right)^{2}+L_{5} r_{0}^{2}+\frac{8 L_{3} r_{0}^{2}\left(3-r_{0}^{2}\right)}{\left(1-r_{0}^{2}\right)^{2}}
$$

The proof of this theorem is complete.

Acknowledgements. The research was partly supported by the National Natural Science Foundation of China (No. 11801159). The work of the third author is supported by Mathematical Research Impact Centric Support (MATRICS) of the Department of Science and Technology (DST), India (MTR/2017/000367).

Conflict of Interests. The authors declare that there is no conflict of interests regarding the publication of this paper.

Data availability statement. I confirm that there is no data involved in this manuscript.

\section{REFERENCES}

1. Z. Abdulhadi and Y. Muhanna, Landau's theorem for biharmonic mappings, J. Math. Anal. Appl. 338 (2008), 705-709.

2. H. Begehr, Boundary value problem in complex analysis II, Bol. Asoc. Mat. Venezolana, 2 (2005), 217-250.

3. H. Begehr and T. Vaitekhovich, Complex partial differential equations in a manner of I. N. Vekua, Lect. Notes TICMI, 8 (2007), 15-26.

4. R. Brody, Compact manifolds and hyperbolicity, Trans. Amer. Math. Soc., 235 (1978), 213219.

5. H. Chen, P. Gauthier and W. Hengartner, Bloch constants for planar harmonic mappings, Proc. Amer. Math. Soc. 128 (2000), 3231-3240.

6. H. Chen, P. Gauthier and W. Hengartner, Bloch constants in several variables, Trans. Amer. Math. Soc. 353 (2001), 1371-1386. 
7. SH. Chen and D. Kalaj, The Schwarz type lemmas and the Landau type theorem of mappings satisfying Poisson's equations, Complex Anal. Oper. Theory 13 (2019), 2049-2068.

8. SH. Chen, P. Li and X. Wang, Schwarz-type lemma, Landau-type theorem, and Lipschitztype space of solutions to inhomogeneous biharmonic equations, J. Geom. Anal., 29 (2019), 2469-2491.

9. SH. Chen, S. Ponnusamy and X. Wang, Landau's theorem for certain biharmonic mappings, Appl. Math. Comput., 208 (2009), 427-433.

10. SH. Chen, S. Ponnusamy and X. Wang, Compositions of harmonic mappings and biharmonic mappings, Bull. Belg. Math. Soc. Simon Stevin, 17 (2010), 693-704.

11. SH. Chen, S. Ponnusamy and X. Wang, Bloch constant and Landau's theorem for planar p-harmonic mappings, J. Math. Anal. Appl., 373 (2011), 102-110.

12. SH. Chen and M. Vuorinen, Some properties of a class of elliptic partial differential operators, J. Math. Anal. Appl., 431 (2015), 1124-1137.

13. SH. Chen and J. Zhu, Schwarz type lemmas and a Landau type theorem of functions satisfying the biharmonic equation, Bull. Sci. Math., 154 (2019), 36-63.

14. F. Colonna, The Bloch constant of bounded harmonic mappings, Indiana Univ. Math. J. 38 (1989), 829-840.

15. P. Gauthier and M. Pouryayevali, Failure of Landau's theorem for quasiconformal mappings of the disc, Contemporay Math., 355 (2004), 265-268.

16. E. Heinz, On one-to-one harmonic mappings, Pacific J. Math. 9 (1959), 101-105.

17. D. Kalaj and M. Pavlović, On quasiconformal self-mappings of the unit disk satisfying Poisson's equation, Trans. Amer. Math. Soc., 363 (2011), 4043-4061.

18. E. Landau, Über die Blochsche konstante und zwei verwandte weltkonstanten, Math. Zeit., 30 (1929), 608-634.

19. P. Li and S. Ponnusamy, Bi-Lipschitz continuity of quasiconformal solutions to the DirichletNeumann problems in the unit disk, Preprint.

20. P. Li, A. Rasila and Z. Wang, On properties of solutions to the $\alpha$-harmonic equation, Complex Var. Elliptic Equ., https://doi.org/10.1080/17476933.2019.1684479.

21. P. Li, X. Wang and Q. Xiao, Several properties of $\alpha$-harmonic functions in the unit disk, Monatsh. Math. 184 (2017), 627-640.

22. M. Pavlović, Introduction to function spaces on the disk, Matematički institut SANU, Belgrade, 2004.

23. H. Wu, Normal families of holomorphic mappings, Acta Math., 119 (1967), 193-233.

24. L. Zalcman, Normal families: New perspectives, Bull. Amer. Math. Soc., 35 (1998), 215-230.

Department of Mathematics, Hunan First Normal University, Changsha, Hunan 410205, People's Republic of ChinA

Email address: wokeyi990163.com

Department of Mathematics, Hunan First Normal University, Changsha, Hunan 410205, People's Republic of China

Email address: luoqh207@qq.com

S. Ponnusamy, Department of Mathematics, Indian Institute of Technology Madras, Chennai-600 036, IndiA.

Email address: samy@iitm.ac.in 